\title{
Physician Care Coordination and the Use of Psychotropic Polypharmacy in the Management of Pediatric Mental Disorders
}

\author{
Rohan Medhekar, PhD; Kayo Fujimoto, PhD; Rajender R. Aparasu, PhD, FAPhA; \\ Vinod S. Bhatara, MD; Michael L. Johnson, PhD; Joy P. Alonzo, RPh, MEng, PharmD; \\ Heidi L. Schwarzwald, MD, MPH; and Hua Chen, MD, PhD
}

\begin{abstract}
BACKGROUND: Psychotropic polypharmacy is a concern in the management of pediatric mental disorders due to the lack of pediatric data to support the practice. Although seeing multiple providers has been identified as an important predictor of polypharmacy, no study has yet assessed the effect of care coordination between providers on receipt of psychotropic polypharmacy.
\end{abstract}

OBJECTIVE: To examine the association between the intensity of care coordination within a patient's care team and the likelihood of the patient receiving multiclass psychotropic polypharmacy.

METHODS: A retrospective study was conducted using the 2013-2015 administrative claims data from a Medicaid managed care organization (Texas Children's Health Plan). Children and adolescents aged 18 years or younger with a diagnosis of a mental/behavioral disorder and receipt of psychotropic prescriptions from multiple prescribers were included in the study. Psychotropic polypharmacy was defined as the receipt of 2 or more psychotropic medications from different drug classes concurrently for 60 days or more. Care coordination was measured using social network analysis (SNA), a new technique included in the Agency for Healthcare Research and Quality Care Coordination Measures Atlas. Care density, an SNA surrogate for care coordination, was calculated as the ratio of the sum of patients shared by physician pairs within a patient's care team to the total number of physician pairs. The Andersen behavioral model was used to guide multivariate logistic regression analyses conducted to assess the association between care density and the likelihood of patients receiving psychotropic polypharmacy after controlling for predisposing and need factors.

RESULTS: A total of 24,147 children and adolescents diagnosed with a mental/ behavioral disorder were identified. About $34.0 \%(n=8,092)$ of these individuals received psychotropic medications from multiple prescribers who were either primary care physicians (PCPs) or specialists. Logistic regression analysis showed a significant association between care density and the use of psychotropic polypharmacy. However, the direction of this relationship varied depending on the composition of the patient's care team. Among patients with only PCPs involved in their care team, patients in the higher care-density group were $28 \%$ less likely to receive psychotropic polypharmacy $(0 \mathrm{R}=0.72 ; 95 \% \mathrm{Cl}=0.62-0.96)$ than those in the lower care-density group. In contrast, among patients who had both PCPs and specialists involved in their care team, those in the higher care-density group were 2 times more likely to experience psychotropic polypharmacy $(0 R=2.01$; $95 \% \mathrm{Cl}=1.68-2.40)$. Care density was not significantly associated with the receipt of psychotropic polypharmacy in the specialist-only group.

CONCLUSIONS: This study found significant associations between care density and prescription of psychotropic polypharmacy. This relationship varied depending on the patient's diagnosis, disease complexity, and composition of the patient's care team.

J Manag Care Spec Pharm. 2019;25(1):29-36

Copyright $\odot 2019$, Academy of Managed Care Pharmacy. All rights reserved.

\section{What is already known about this subject}

Psychotropic polypharmacy is a main safety concern in the management of pediatric mental disorders

Previous studies have shown that involvement of multiple physicians in the provision of care is significantly associated with the receipt of polypharmacy.

\section{What this study adds}

The relationship between care coordination and psychotropic polypharmacy varied depending on the composition of the patient's care team.

Higher care density was associated with lower likelihood of psychotropic polypharmacy among patients treated by primary care physicians (PCPs) and higher likelihood among patients treated by a mix of PCPs and psychiatric specialists.

$\mathrm{P}$ olypharmacy is defined as the use of multiple medications concurrently by a single patient that is clinically indicated. ${ }^{1}$ There is no standard cutoff on the number of medications for the definition of polypharmacy, and researchers have arbitrarily chosen various cutoffs to operationalize it. ${ }^{2}$ Polypharmacy can be classified as within-class polypharmacy (i.e., concurrent use of multiple medications from the same drug class) or multiclass polypharmacy (i.e., concurrent use of multiple medications from different drug classes for the same symptom cluster). ${ }^{1}$

Psychotropic polypharmacy is the practice of polypharmacy in psychiatric therapy. The most commonly used definition of psychotropic polypharmacy is the use of 2 or more psychotropic medications in the same patient or the use of 2 or more psychotropic medications to treat the same condition., ${ }^{1,3}$ Despite the recommendations of clinical guidelines for the use of monotherapy in the treatment of children and adolescents with mental/behavioral disorders, the use of polypharmacy is quite common in psychiatric care, with the overall prevalence ranging from $14 \%-73 \% .^{4,5-12}$ Psychotropic polypharmacy is a public health concern because of limited scientific evidence for understanding the immediate and/or long-term effects of its use on children and adolescents. ${ }^{13-16}$ Additionally, psychotropic polypharmacy has been associated with a number of negative 
consequences, including adverse events, drug-drug interactions, nonadherence, higher health care costs, morbidity, and mortality. $3,13-19$

Previous studies have shown that involvement of multiple physicians in the provision of care is significantly associated with the receipt of polypharmacy. ${ }^{20,21}$ Fragmented care and lack of coordination among the physicians on a patient's care team can lead to continuation of psychotropic polypharmacy in cases where it is not medically essential. ${ }^{22}$ Recently, the concepts and techniques of social network analysis have been used to characterize the professional relationships among providers that result from day-to-day interactions, patient referrals, and shared patients. Researchers have used the number of shared patients as an indicator of the strength of provider collaborative relationships. Pollack et al. (2012) developed a measure called care density, which determines the extent of patient sharing among physicians. ${ }^{23}$ It is hypothesized that providers who share greater numbers of patients have stronger collaborative relationships and will be able to provide better-coordinated care. A study by Barnett et al. (2011) showed that physicians who shared 8 or more patients had $80 \%$ probability of having an information-sharing relationship. ${ }^{24}$ Several studies have demonstrated a direct association between care density and health care outcomes such as quality of care, cost of care, and hospital outcomes. ${ }^{23,25,26}$ However, to our knowledge, there has been no study that has looked at the relationship between care density and psychotropic polypharmacy.

Therefore, the objective of this study was to examine the relationship between care density and receipt of multiclass psychotropic polypharmacy among children and adolescents with mental/behavioral disorders. Our hypothesis was that the higher the care density, the lower the probability of receiving psychotropic polypharmacy would be. Further, we examined if this relationship varied depending on the specialty of physicians involved in the care team.

\section{Methods}

\section{Study Design and Data Source}

A retrospective study was conducted using administrative claims data from Texas Children's Health Plan (TCHP) for the period July 1, 2013-June 30, 2015. Data were obtained for children and adolescents who were ever diagnosed with mental/behavioral disorders and identified using International Classification of Diseases, Ninth Revision, Clinical Modification (ICD-9-CM) codes. TCHP is a pediatric Medicaid managed care program that covers more than 450,000 members aged 18 years or under. There are over 1,100 primary care physicians (PCPs), 3,200 specialists, and 60 hospitals that provide service and patient care to these members. The data were deidentified in accordance with Health Insurance Portability and Accountability Act standards.

\section{Study Sample}

Patients were identified if they (a) were aged $\leq 18$ years, (b) were diagnosed with a mental/behavioral disorder (identified using ICD-9-CM codes; see Appendix A, available in online article), (c) had at least 1 pharmacy claim of psychotropic medication, and (d) received a prescription for psychotropic medication from multiple (at least 2 ) prescribers during the study period. Further, these individuals were required to be continuously enrolled in the health plan throughout the study period. The psychotropic medications considered in the study are listed in Appendix B (available in online article).

\section{Outcome Measure}

Prescription fills for psychotropic medications, including fill date and days supply, were obtained from the pharmacy component of TCHP data. The outcome of interest was whether an individual had at least 1 episode of multiclass psychotropic polypharmacy during the study period, measured as a binary variable (1: Yes, 0: No). An episode of multiclass psychotropic polypharmacy was defined as overlapping fills of $\geq 2$ psychotropic medications from different drug classes for 60 days or more, with no gaps in polypharmacy treatment. ${ }^{5}$ The 60-day overlap criterion is the most commonly implemented cutoff used to define polypharmacy because it avoids misclassifying instances of cross-titration as polypharmacy. ${ }^{27,28}$ Patients who received psychotropic medications but did not have a polypharmacy episode at any time during the study period were classified as nonpolypharmacy cases. Episodes of treatment were identified using the prescription fill date and the days supply information available from the pharmacy claims. Before measuring the episodes, overlapping days supply for the same medication was carried forward, assuming that the patient finished the current prescription before starting on the refill prescription. Gaps in fills of the same medication of $\leq 15$ days were allowed and adjusted in the calculation of the overlap. Only unique combinations of drug classes of at least 60 days were considered.

\section{Exposure Measures}

The Andersen behavioral model was used to guide the selection of the potential predictors that could help explain the variation in the receipt of psychotropic polypharmacy. ${ }^{29}$ The components of the model, including predisposing, enabling, and need factors, were defined based on literature and relevance to the study's objective. Predisposing factors included patient's age, sex, and race/ethnicity. A patient's care team density was considered as the enabling factor. Need factors included the number of mental/behavioral disorders diagnosed, the type of mental/behavioral disorder diagnosed, and the number of prescribers involved in treatment.

Care density (enabling factor) was the primary independent variable of the study. To measure care density, a physician 
patient-sharing network was first constructed using pharmacy claims data. A pair of physicians was considered to have shared a patient if they both prescribed medications to a given patient any time during the study period. A tie formed through such patient-sharing was called a network tie. A set of all such possible ties between all the physicians in the data formed the physician patient-sharing network. The number of patients shared between physicians was used to characterize the collaborative relationships between physicians. ${ }^{23-26}$

The extent of patient-sharing among physicians was estimated using the care-density measure developed by Pollack et al. ${ }^{23}$ Care density is a patient-level measure, calculated as the ratio of the sum of patients shared by physician pairs within a patient's care team to the total number of physician pairs within the patient's care team..$^{23}$ Care density corresponds to the care team's cohesiveness, which is theoretically a representation of better communication and information-sharing between the patient's care team. ${ }^{25}$ A greater care-density value indicates stronger cohesiveness among the care team. ${ }^{26}$ Care density had a highly skewed distribution in this study.

For the care teams that involved both PCPs and specialists, the $10 \%, 25 \%, 50 \%, 75 \%$, and $90 \%$ quantiles were $1,1.7,5.7$, 36.3, and 144.7, respectively. Between PCPs and specialists, those who ever shared 1 patient (10\% and $25 \%$ quantiles) and those who never shared a patient probably do not have much implication on the relationship between the 2 providers. Similarly, sharing 145 patients may not be much different than sharing 37 patients. A previous study by Blanchet et al. (2011) found that pairs of physicians in a single academic health care system who shared $\geq 8$ patients had an $80 \%$ probability of having a validated information-sharing relationship. ${ }^{30}$ In this study, the median care density was closest to this validated cutoff, so care density was operationalized as a binary variable $(0=$ care density less than the median; $1=$ care density greater than or equal to the median). ${ }^{26}$

Considering that patients with differing disease complexities might be treated by care teams consisting of various types of providers (e.g., PCPs only, or combination of PCPs and psychiatric specialists, or multiple specialists) and that the need and the purpose of care coordination-and the actual information exchanged-might differ between PCP-PCP, PCPspecialist, or specialist-specialist, stratification by physician specialty was necessary. To understand the variant implications of care density on psychotropic polypharmacy in different types of patient care teams, 3 patient groups were created based on the type of physicians involved in the care team: (a) PCPs only (e.g., general medicine, family medicine, and internal medicine); (b) specialists only (e.g., psychiatry, addiction medicine, and psychosomatic medicine); and (c) PCPs and specialists.
Patients who were prescribed psychotropic medications by physicians of any other specialty than those previously listed were excluded from the study. The prescribing physician and his/her specialty were identified using the National Provider Identifier (NPI) number and the physician specialty reported in the pharmacy claims component of the TCHP data. The National Plan and Provider Enumeration System NPI database was used to obtain information on specialty for physicians with missing values for specialty in the TCHP data.

\section{Statistical Analysis}

Descriptive statistics, including frequencies, means ( \pm standard deviation $[S D]$ ), and medians, were used to characterize patient demographics, clinical characteristics, and care density. Separate logistic regression models were fitted for patients with different types of care teams to determine associations between care density and the receipt of multiclass psychotropic polypharmacy after controlling for predisposing and need factors.

A priori significance level of $P<0.05$ was chosen for the analyses. All analyses were conducted by using SAS 9.3 software (SAS Institute, Cary, NC). The study was reviewed and approved by the University of Houston Institutional Review Board.

\section{Results}

\section{Patient Characteristics}

A total of 24,147 children and adolescents were diagnosed with a mental/behavioral disorder and had at least 1 pharmacy claim of psychotropic medication during the study period. About 34\% $(n=8,092)$ of these individuals received these prescriptions from multiple prescribers who were either PCPs or specialists. According to the types of providers involved in prescribing, these patients were divided into the following 3 groups: PCPs only ( $\mathrm{n}=3,408 ; 42.1 \%)$, specialists only $(n=1,921 ; 23.7 \%)$, and PCPs and specialists ( $n=2,763 ; 34.1 \%)$. About $29 \%(n=2,371)$ of the study population received polypharmacy. Most patients in the study were male (65\%); 36\% were Caucasians and 38\% were Hispanics; and 70\% were between the ages of 4 and 12 years.

Receipt of Psychotropic Polypharmacy. The PCP + specialist group was the group that had the highest utilization rate of psychotropic polypharmacy (42.9\%), followed by the specialistonly group (40.5\%), with the lowest observed among the PCPonly group (12.0\%; Table 1$)$.

Predisposing Characteristics. Within the PCP-only group, a significantly higher proportion of males received polypharmacy $(74 \%)$ compared with females $(P=0.02)$; however, there was no statistically significant difference within other groups among males and females with respect to receipt of polypharmacy. African American and Hispanic patients were less likely to receive polypharmacy across all study groups, whereas Caucasians were more likely to receive polypharmacy $(P<0.05$; 
TABLE 1 Demographic and Clinical Characteristics of Patients Stratified by Receipt of Polypharmacy

Multiple Prescribers Involved in Treatment $(\mathrm{N}=8,092)$

\begin{tabular}{|c|c|c|c|c|c|c|c|c|c|}
\hline \multirow[b]{3}{*}{ Characteristic } & \\
\hline & \multicolumn{3}{|c|}{ PCPs Only (n=3,408; 42.1\%) } & \multicolumn{3}{|c|}{ Specialists Only $(\mathrm{n}=1,921 ; 23.7 \%)$} & \multicolumn{3}{|c|}{ PCPs + Specialists $(\mathrm{n}=2,763 ; 34.1 \%)$} \\
\hline & $\begin{array}{c}\text { No } \\
\text { Polypharmacy } \\
(\mathbf{n}=3,000 ; \\
88.0 \%)\end{array}$ & $\begin{array}{c}\text { Psychotropic } \\
\text { Polypharmacy } \\
(\mathrm{n}=408 \\
12.0 \%) \\
\end{array}$ & $P$ Value & \begin{tabular}{|c|} 
No \\
Polypharmacy \\
$(\mathrm{n}=1,143$ \\
$59.5 \%)$
\end{tabular} & $\begin{array}{c}\text { Psychotropic } \\
\text { Polypharmacy } \\
\text { (n =778; } \\
40.5 \%)\end{array}$ & $P$ Value & \begin{tabular}{|c|} 
No \\
Polypharmacy \\
$(\mathrm{n}=1,578$ \\
$57.1 \%)$ \\
\end{tabular} & $\begin{array}{c}\text { Psychotropic } \\
\text { Polypharmacy } \\
\quad(n=1,185 \\
42.9 \%)\end{array}$ & $P$ Value \\
\hline \multicolumn{10}{|l|}{ Predisposing characteristics } \\
\hline \multicolumn{10}{|l|}{ Age group, years, $\mathrm{n}(\%)$} \\
\hline $0-3$ & $43 \quad(1.4)$ & $3(0.7)$ & & $8 \quad(0.7)$ & $7 \quad(0.9)$ & & $10 \quad(0.6)$ & $11 \quad(0.9)$ & \\
\hline $4-8$ & $1,172(39.1)$ & $176(43.1)$ & & $380(33.3)$ & $216(27.8)$ & & $625(39.6)$ & $452(38.1)$ & \\
\hline $9-12$ & $1,182(39.4)$ & $180(44.1)$ & & $344(30.1)$ & $249(32.0)$ & & $516(32.7)$ & $407(34.4)$ & \\
\hline $13-18$ & $603(20.1)$ & $49(12.0)$ & & $411(36.0)$ & $306(39.3)$ & & $427(27.1)$ & $315(26.6)$ & \\
\hline Mean $( \pm S D)^{a}$ & $9.62 \pm 3.25$ & $9.14 \pm 2.78$ & $<0.001$ & $10.61 \pm 3.66$ & $10.92 \pm 3.59$ & 0.535 & $9.96 \pm 3.55$ & $9.88 \pm 3.43$ & 0.213 \\
\hline \multicolumn{10}{|l|}{ Sex, n (\%) } \\
\hline Male & $2,064(68.8)$ & $303(74.3)$ & 0.025 & $670(58.7)$ & $465(59.8)$ & 0.637 & $1,023(64.8)$ & $781(65.9)$ & 0.572 \\
\hline \multicolumn{10}{|l|}{ Race/ethnicity, n (\%) } \\
\hline African American & $621(20.7)$ & $68(16.7)$ & 0.057 & $286(25.0)$ & $191(24.6)$ & 0.829 & $406(25.7)$ & $239(20.2)$ & $<0.001$ \\
\hline Caucasian & $1,144(38.1)$ & $227(55.6)$ & $<0.010$ & $239(20.9)$ & $260(33.4)$ & $<0.001$ & $452(28.6)$ & $584(49.3)$ & $<0.001$ \\
\hline Hispanic & $1,095(36.5)$ & $105(25.7)$ & $<0.001$ & $573(50.1)$ & $297(38.2)$ & $<0.001$ & $672(42.6)$ & $324(27.3)$ & $<0.001$ \\
\hline Not reported & $140 \quad(4.7)$ & $8 \quad(2.0)$ & 0.068 & $45 \quad(3.9)$ & $30 \quad(3.9)$ & 0.878 & $48 \quad(3.0)$ & $38 \quad(3.2)$ & 0.896 \\
\hline \multicolumn{10}{|l|}{ Enabling characteristics } \\
\hline \multicolumn{10}{|l|}{ Care density } \\
\hline Mean $( \pm$ SD $)$ & $25.40 \pm 31.90$ & $21.15 \pm 24.32$ & $<0.001$ & $43.90 \pm 79.66$ & $29.72 \pm 52.83$ & 0.119 & $26.48 \pm 51.86$ & $44.43 \pm 59.44$ & $<0.001$ \\
\hline Median & 14.00 & 11.00 & $<0.001$ & 9.00 & 7.42 & 0.278 & 3.58 & 11.50 & $<0.001$ \\
\hline \multicolumn{10}{|l|}{ Need characteristics } \\
\hline \multicolumn{10}{|c|}{ Number of mental/behavioral disorders diagnosed, n (\%) } \\
\hline 0 & $47(1.6)$ & $5(1.2)$ & & $1 \quad(0.1)$ & $0 \quad(0)$ & & $14 \quad(0.9)$ & $3(0.3)$ & \\
\hline 1 & $2,002(66.7)$ & $172(42.2)$ & & $367(32.1)$ & $118(15.2)$ & & $576(36.5)$ & $306(25.8)$ & \\
\hline $2-4$ & $922(30.7)$ & $209(51.2)$ & & $686(60.0)$ & $513(65.9)$ & & $897(56.8)$ & $710(59.9)$ & \\
\hline$\geq 5$ & $29(1.0)$ & $22 \quad(5.4)$ & & $89(7.8)$ & $147(18.9)$ & & $91 \quad(5.8)$ & $166(14.0)$ & \\
\hline Mean $( \pm$ SD) & $1.46 \pm 0.84$ & $2.06 \pm 1.25$ & $<0.001$ & $2.37 \pm 1.33$ & $3.14 \pm 1.59$ & 0.894 & $2.18 \pm 1.27$ & $2.71 \pm 1.60$ & 0.043 \\
\hline \multicolumn{10}{|c|}{ Type of mental/behavioral disorder diagnosed, $\mathrm{n}(\%)^{\mathrm{a}}$} \\
\hline ADHD & $2,731(91.0)$ & $390(95.6)$ & $<0.001$ & $843(73.8)$ & $588(75.6)$ & 0.393 & $1,298(82.3)$ & $1,031(87.0)$ & $<0.001$ \\
\hline Bipolar disorders & $79 \quad(2.6)$ & $32(7.8)$ & $<0.001$ & $325(28.4)$ & $473(60.8)$ & $<0.001$ & $295(18.7)$ & $481(40.6)$ & $<0.001$ \\
\hline Depression & $156 \quad(5.2)$ & $44(10.8)$ & $<0.001$ & $317(27.7)$ & $291(37.4)$ & $<0.001$ & $330(20.9)$ & $299(25.2)$ & $<0.001$ \\
\hline Anxiety & $185 \quad(6.2)$ & $63(15.4)$ & $<0.001$ & $214(18.7)$ & $216(27.8)$ & $<0.001$ & $276(17.5)$ & $253(21.4)$ & 0.011 \\
\hline Learning disorders & $308(10.3)$ & $49(12.0)$ & 0.301 & $126(11.0)$ & $71 \quad(9.1)$ & 0.193 & $190(12.0)$ & $112 \quad(9.5)$ & 0.031 \\
\hline Adjustment disorders & $218 \quad(7.3)$ & $37 \quad(9.1)$ & 0.192 & $186(16.3)$ & $144(18.5)$ & 0.218 & $207(13.1)$ & $192(16.2)$ & 0.031 \\
\hline Conduct disorder & $275 \quad(9.2)$ & $53(13.0)$ & 0.019 & $166(14.5)$ & $134(17.2)$ & 0.110 & $250(15.8)$ & $190(16.0)$ & 0.916 \\
\hline Oppositional defiant disorder & $120 \quad(4.0)$ & $75(18.4)$ & $<0.001$ & $219(19.2)$ & $198(25.5)$ & 0.001 & $296(18.8)$ & $295(24.9)$ & $<0.010$ \\
\hline Schizophrenia & $35(1.2)$ & 17 (4.2) & $<0.001$ & $86 \quad(7.5)$ & $127(16.3)$ & $<0.001$ & $81 \quad(5.1)$ & $117 \quad(9.9)$ & $<0.010$ \\
\hline \multicolumn{10}{|c|}{ Number of prescribers involved in treatment, $\mathbf{n}(\%)$} \\
\hline $2-4$ & $2,841(94.7)$ & $376(92.2)$ & & $1,126(98.5)$ & $732(94.1)$ & & $1,479(93.7)$ & $978(82.5)$ & \\
\hline$\geq 5$ & $159 \quad(5.3)$ & $32(7.8)$ & & $17 \quad(1.5)$ & $46 \quad(5.9)$ & & $99 \quad(6.3)$ & $207(17.5)$ & \\
\hline Mean $( \pm S D)$ & $2.59 \pm 1.03$ & $2.84 \pm 1.38$ & $<0.001$ & $2.29 \pm 0.63$ & $2.67 \pm 1.03$ & $<0.001$ & $2.73 \pm 1.02$ & $3.40 \pm 1.34$ & $<0.001$ \\
\hline \multicolumn{10}{|c|}{ Hospitalization/ED visit, n (\%) } \\
\hline Yes & $238 \quad(7.9)$ & $44(10.8)$ & 0.050 & $297(26.0)$ & $316(40.6)$ & $<0.001$ & $290(18.4)$ & $350(29.5)$ & $<0.001$ \\
\hline
\end{tabular}

chi-square test between those who received polypharmacy vs. those who did not receive polypharmacy within each group).

Need Characteristics. Within the PCP-only group and the $\mathrm{PCP}+$ specialist group, patients who received polypharmacy had a significantly higher number of mental/behavioral disorders (t-test, $P<0.05$ ); however, this was not observed in the specialist-only group. Across all study groups, the mean number of prescribers involved in treatment was higher in those 
TABLE 2 Multivariate Logistic Regression Model by Study Groups

\begin{tabular}{|c|c|c|c|c|c|c|c|c|c|}
\hline \multirow[b]{2}{*}{ Characteristic } & \multicolumn{3}{|c|}{ PCPs Only } & \multicolumn{3}{|c|}{ Specialists Only } & \multicolumn{3}{|c|}{ PCPs + Specialists } \\
\hline & OR & $95 \% \mathrm{CI}$ & $P$ Value & OR & $95 \% \mathrm{CI}$ & $P$ Value & OR & $95 \% \mathrm{CI}$ & $P$ Value \\
\hline \multicolumn{10}{|l|}{ Enabling characteristics } \\
\hline \multicolumn{10}{|c|}{ Care density (ref: less than median care density) } \\
\hline Cared density (higher than median) & 0.72 & $0.62-0.96$ & 0.022 & 0.93 & $0.75-1.15$ & 0.499 & 2.01 & $1.68-2.40$ & $<0.001$ \\
\hline \multicolumn{10}{|l|}{ Predisposing characteristics } \\
\hline Age & 0.96 & $0.93-1.00$ & 0.050 & 1.01 & $0.97-1.04$ & 0.780 & 0.97 & $0.94-0.99$ & 0.018 \\
\hline \multicolumn{10}{|l|}{ Sex (ref: female) } \\
\hline Male & 1.46 & $1.14-1.89$ & 0.003 & 1.17 & $0.94-1.46$ & 0.171 & 1.17 & $0.98-1.42$ & 0.091 \\
\hline \multicolumn{10}{|l|}{ Race/ethnicity (ref: African American) } \\
\hline Caucasian & 1.82 & $1.34-2.46$ & $<0.001$ & 1.48 & $1.11-1.96$ & 0.007 & 2.19 & $1.75-2.73$ & $<0.001$ \\
\hline Hispanic & 0.93 & $0.66-1.30$ & 0.656 & 0.80 & $0.62-1.03$ & 0.087 & 0.89 & $0.71-1.12$ & 0.317 \\
\hline Not reported & 0.57 & $0.26-1.24$ & 0.156 & 1.02 & $0.59-1.75$ & 0.951 & 1.60 & $0.98-2.62$ & 0.061 \\
\hline \multicolumn{10}{|l|}{ Need characteristics } \\
\hline $\begin{array}{l}\text { Number of mental health disorders } \\
\text { diagnosed }\end{array}$ & 1.88 & $1.38-2.57$ & $<0.001$ & 0.91 & $0.70-1.19$ & 0.488 & 1.35 & $1.05-1.72$ & 0.018 \\
\hline \multicolumn{10}{|c|}{ Type of mental/behavioral disorder diagnosed (ref: no) } \\
\hline ADHD & 1.78 & $0.95-3.33$ & 0.070 & 2.49 & $1.67-3.70$ & $<0.001$ & 1.33 & $0.93-1.92$ & 0.119 \\
\hline Bipolar disorders & 1.51 & $0.85-2.68$ & 0.163 & 4.36 & $3.04-6.26$ & $<0.001$ & 2.12 & $1.52-2.95$ & $<0.001$ \\
\hline Depression & 1.06 & $0.61-1.83$ & 0.838 & 1.59 & $1.07-2.36$ & 0.022 & 0.89 & $0.62-1.28$ & 0.529 \\
\hline Anxiety & 1.43 & $0.88-2.33$ & 0.151 & 1.63 & $1.11-2.40$ & 0.013 & 1.00 & $0.71-1.41$ & 0.992 \\
\hline Learning disorders & 0.42 & $0.26-0.70$ & $<0.001$ & 1.17 & $0.74-1.83$ & 0.505 & 0.51 & $0.35-0.75$ & $<0.001$ \\
\hline Adjustment disorders & 0.39 & $0.23-0.68$ & $<0.001$ & 1.04 & $0.72-1.51$ & 0.842 & 0.68 & $0.47-0.97$ & 0.032 \\
\hline Conduct disorder & 0.55 & $0.34-0.89$ & 0.015 & 0.95 & $0.63-1.43$ & 0.789 & 0.60 & $0.42-0.85$ & 0.004 \\
\hline Oppositional defiant disorder & 2.13 & $1.33-3.40$ & 0.002 & 1.20 & $0.83-1.75$ & 0.341 & 0.81 & $0.58-1.14$ & 0.227 \\
\hline Schizophrenia & 1.78 & $0.86-3.68$ & 0.123 & 1.66 & $1.07-2.56$ & 0.023 & 1.00 & $0.64-1.56$ & 0.982 \\
\hline \multicolumn{10}{|l|}{ Hospitalization/ED visit (ref: no) } \\
\hline Yes & 1.08 & $0.74-1.58$ & 0.677 & 0.95 & $0.71-1.26$ & 0.702 & 1.12 & $0.89-1.41$ & 0.316 \\
\hline $\begin{array}{l}\text { Number of prescribers involved } \\
\text { in treatment }\end{array}$ & 1.12 & $1.02-1.23$ & 0.014 & 1.68 & $1.46-1.92$ & $<0.001$ & 1.40 & $1.29-1.51$ & $<0.001$ \\
\hline
\end{tabular}

who received polypharmacy $(P<0.001)$. Similarly, across all study groups, those who received polypharmacy had a higher number of emergency department (ED) visits/hospitalizations compared with those who did not receive polypharmacy $(P<0.05$, chi-square test within each group).

Enabling Factors. Unlike the consistent differences observed on predisposing and need factors between polypharmacy recipients and nonrecipients across the study groups, the descriptive statistics of care density to receipt of psychotropic polypharmacy varied significantly across the PCP-only study group and $\mathrm{PCP}+$ specialist group $(P<0.001$; Table 1$)$. Median care density was higher among nonpolypharmacy recipients compared with polypharmacy recipients among the PCP-only and specialist-only groups. In contrast, median care density was lower in nonpolypharmacy recipients compared with polypharmacy recipients in the $\mathrm{PCP}+$ specialist group.

\section{Multivariable Logistic Regression}

Predisposing Characteristics. In all groups, Caucasians had a higher likelihood of receiving psychotropic polypharmacy than
African Americans. The direction of the effect estimates for age, race/ethnicity, and gender were consistent across study groups. However, statistically significant differences were only detected among some provider groups. For instance, males had a $46 \%$ higher probability of receiving psychotropic polypharmacy than females in the PCP-only group (odds ratio $[\mathrm{OR}]=1.46$; $95 \%$ confidence interval $[\mathrm{CI}]=1.14-1.89$ ), while the association between gender and receipt of polypharmacy in the PCP + specialist group was not statistically significant $(\mathrm{OR}=1.17 ; 95 \%$ $\mathrm{CI}=0.98-1.42 ;$ Table 2).

Need Characteristics. An increase in the number of prescribers involved in treatment was associated with a higher likelihood of receiving psychotropic polypharmacy among all groups. An increase in the number of diagnosed mental disorders was associated with a higher likelihood of receiving psychotropic polypharmacy in the PCP-only and PCP + specialist groups (Table 2).

Enabling Characteristics. For the PCP-only group, patients in the high care-density group were $28 \%$ less likely to receive 
psychotropic polypharmacy $(\mathrm{OR}=0.72 ; 95 \% \mathrm{CI}=0.62-0.96)$. For the specialist-only group, care density was not significantly associated with receipt of psychotropic polypharmacy among patients who received psychotropic prescriptions from multiple specialists. In the PCP + specialist group, patients in the higher care-density group were 2 times more likely to experience psychotropic polypharmacy $(\mathrm{OR}=2.01 ; 95 \% \mathrm{CI}=1.68-2.40)$ than patients with lower care density (Table 2).

\section{Discussion}

This is the first study to examine the association between physician patient-sharing relationships and prescription of psychotropic polypharmacy using the concepts of social network analysis. ${ }^{25}$ The primary finding of our study is that the enabling factor representing the extent of patient-sharing between the patient's care team (i.e., care density) was strongly associated with the likelihood of receiving psychotropic polypharmacy. However, the relationship between care density and psychotropic polypharmacy varied depending on the specialty of physicians involved in the care team. It was observed that, among patients with only PCPs involved in the care team, higher care density was associated with lower likelihood of receiving psychotropic polypharmacy. On the other hand, in the group of patients who had both PCPs and specialists, higher care density was associated with higher likelihood of receiving psychotropic polypharmacy.

The various implications of care density in patient groups seen by different care teams could be because the study groups represent patients of differing diagnoses and clinical complexities and, therefore, have differing needs for psychotropic polypharmacy. When the care team consists of PCPs only, nearly $90 \%$ of patients had a diagnosis of attention-deficit/hyperactivity disorder (ADHD) only. The clinical practice guidelines for diagnosis and treatment of ADHD recommend the use of either behavioral therapy, medication, or a combination of behavioral therapy and medication for the treatment of uncomplicated ADHD. ${ }^{31}$ Combination or augmentation therapy is not recommended for management of ADHD unless the patient is diagnosed with comorbid conditions such as bipolar disorders, tics, or anxiety disorders, in which case, short-term polypharmacy might be used. ${ }^{32}$ Moreover, seeing multiple PCPs does not suggest increased clinical complexity in our study. It rather implies that patients either changed their PCP or received prescriptions from multiple PCPs from a shared practice.

The patient group who was seen by multiple mental health specialists is distinctively different from the PCP-only group. Children in this group had a much higher prevalence of mood disorders-especially bipolar disorder and depression-compared with the PCP-only group. Evidence-based studies have shown that polypharmacy is more effective than monotherapy in some patients with mood disorder. A combination of an atypical antipsychotic and a mood stabilizer for acute mania and a combination of an atypical antipsychotic and an antidepressant for treatment-resistant depression have been approved by the U.S. Food and Drug Administration. ${ }^{33,34}$ Treatment guidelines for children and adolescents with bipolar disorders also recommend the use of augmented therapy when monotherapy does not work. ${ }^{35}$ Moreover, this group is also the most severe subgroup with respect to clinical complexity. About 32\% of children and adolescents in this subgroup were either hospitalized or had an ED visit, and about 75\% had multiple comorbid psychiatric disorders. Therefore, children and adolescents in this group might be in need of intensive pharmacotherapy including psychotropic polypharmacy.

The patients who received psychotropic prescriptions from both PCPs and specialists had a diagnostic profile and clinical complexity more similar to patients who received care from multiple specialists than those received care from PCPs only. These patients often are identified by their PCPs or self-identified as needing specialty care, which implies that treatment escalation or adjustment is expected.

Care density is a surrogate measure for care communication and collaboration..$^{23,25}$ It is based on the concept and methods of social network analysis and patient sharing. Networks built on the basis of patient-sharing relationships have been previously validated by Barnett et al. (2011). ${ }^{24}$ Care coordination is defined as "the deliberate organization of patient care activities between 2 or more participants involved in a patient's care to facilitate the appropriate delivery of health care services." ${ }^{\text {"36 }}$ The purpose of collaboration is to better meet the needs of patients, provide sufficient treatment, and reduce redundancy and medication errors. Probably due to the various needs of each patient subgroup on psychotropic polypharmacy, the care collaboration therefore had different implications. Specifically, higher care density or better care coordination lead to less use of psychotropic polypharmacy in patients with uncomplicated ADHD (those who see PCPs only) but increased use among the more severe cases with bipolar disorders or multiple comorbid mental disorders who are seeking specialty care ( $\mathrm{PCP}+$ specialist group). For those clinically complex patients who see multiple specialists, polypharmacy might be justified or might even be a norm; thus, probably, care coordination does not play a major role in prescription of polypharmacy.

Our study suggests that the receipt of psychotropic polypharmacy is not only determined by predisposing and need factors as reported in the previous literature but is also strongly associated with enabling factors such as care collaboration within a patient's care team. ${ }^{27,28,37}$ The findings suggest that, to 
the opposite from the stigma associated with pediatric psychotropic polypharmacy, the practice is mainly resulting from a patient's clinical complexity and improved collaboration within the patient's care team. Psychotropic polypharmacy might be needed in these patients, and the use could be well justified. Appropriate follow-up and monitoring of these patients are all the more important to make sure that psychotropic polypharmacy is effective in reducing symptoms and not leading to unwanted side effects.

Recently, the Care Coordination Measures Atlas Update developed for the Agency for Healthcare Research and Quality elaborated on social network analysis as an emerging method for the measurement of care coordination. ${ }^{38}$ Patient-sharing networks developed using the concepts of social network analysis represent a probable mechanism of care coordination. Early work by Barnett et al. (2011), Pollack et al. (2012, 2015), Ong et al. (2015), and others is promising and has shown that care density is independently associated with various aspects of health care, such as quality of care, prescribing error rates, and costs..$^{23-26}$ Our study adds to this pool of research and advances the understanding of using patient-sharing networks to measure care coordination in mental health care.

\section{Limitations}

There are a few limitations to this study that need to be considered. First, care density was calculated as a claim-based measure of coordination on the assumption that physicians who share more patients are more likely to collaborate; however, whether care was actually coordinated for a given patient could not be directly estimated using the current data. Second, care density might be affected by the structure of health care organizations (size of practice, mode of information exchange), which was not accounted for in this study. Further, withinclass polypharmacy was not considered for this study, although literature suggests that it is highly prevalent $(<1 \%$ of the study population received within-class polypharmacy).

\section{Conclusions}

This study found significant associations between care density and prescription of psychotropic polypharmacy. It was further observed that this relationship varied depending on the composition of the patient's care team. The patient's diagnosis and disease complexity also affected the relationship between care density and psychotropic polypharmacy. Care density as a measure of care coordination is still in its developing stages; however, it might be possible to use care density in combination with other measures of care coordination to completely characterize multiple aspects of coordination.

\section{Authors}

ROHAN MEDHEKAR, PhD; RAJENDER R. APARASU, PhD, FAPhA; MICHAEL L. JOHNSON, PhD; and HUA CHEN, MD, PhD, Department of Pharmaceutical Health Outcomes and Policy, University of Houston College of Pharmacy, Houston, Texas. KAYO FUJIMOTO, PhD, School of Public Health, Center for Health Promotion and Prevention Research, and Center for Infectious Diseases, The University of Texas Health Science Center at Houston. VINOD S. BHATARA, MD, Avera Behavioral Health Center, Sanford School of Medicine, University of South Dakota, Sioux Falls; JOY P. ALONZO, RPh, MEng, PharmD, Rangel College of Pharmacy, Texas A\&M University Health Science Center, Kingsville; and HEIDI L. SCHWARZWALD, MD, MPH, Texas Children's Health Plan and Baylor College of Medicine, Department of Pediatrics, Bellaire, Texas.

AUTHOR CORRESPONDENCE: Hua Chen, MD, PhD, Associate Professor, Department of Pharmaceutical Health Outcomes and Policy, University of Houston College of Pharmacy, Health and Biomedical Sciences Bldg. 2, Rm 4049, 4849 Calhoun Rd., Houston, TX 77204. Tel.: 832.842.8355; E-mail: hchen25@central.uh.edu.

\section{DISCLOSURES}

No outside funding supported this study. The authors do not have any financial relationships or potential conflicts of interest relevant to this article to disclose.

The abstract for part of this study, titled "Association Between Physician Care Coordination and the Use of Psychotropic Polypharmacy in the Management of Pediatric Mental Disorders," was selected as a silver medal abstract and was presented at the AMCP Managed Care \& Specialty Pharmacy Annual Meeting 2017; March 27-30, 2017; Denver, CO.

\section{ACKNOWLEDGMENTS}

Medical writing assistance was provided by Navneet Upadhyay, MS, a graduate student of Department of Pharmaceutical Health Outcomes and Policy, University of Houston College of Pharmacy.

\section{REFERENCES}

1. Shrivastava A, Kukreja S, Kalra G, Shah N. Polypharmacy in psychiatry: a review. Mens Sana Monographs. 2013;11(1):82.

2. Maher R, Hanlon J, Hajjar E. Clinical consequences of polypharmacy in elderly. Expert Opin Drug Saf. 2013;13(1):57-65.

3. National Association of State Mental Health Program Directors, Medical Directors Council. Technical report on psychiatric polypharmacy. October 9 , 2001. Available at: https://www.nasmhpd.org/sites/default/files/ Polypharmacy.pdf. Accessed October 13, 2018.

4. American Academy of Child and Adolescent Psychiatry. Prescribing psychoactive medication for children and adolescents. 2001. Available at: https://www.aacap.org/aacap/policy_statements/2001/Prescribing_ Psychoactive_Medication_for_Children_and_Adolescents.aspx. Accessed October 13, 2018

5. Chen H, Patel A, Sherer J, Aparasu R. The definition and prevalence of pediatric psychotropic polypharmacy. Psychiatr Serv. 2011;62(12):1450-55.

6. Comer J, Olfson M, Mojtabai R. National trends in child and adolescent psychotropic polypharmacy in office-based practice, 1996-2007. J Am Acad Child Adolesc Psychiatry. 2010;49(10):1001-10.

7. McIntyre R, Jerrell J. Polypharmacy in children and adolescents treated for major depressive disorder. J Clin Psychiatry. 2009;70(2):240-46. 
8. Duffy F, Narrow W, Rae D, et al. Concomitant pharmacotherapy among youths treated in routine psychiatric practice. J Child Adolesc Psychopharmacol. 2005;15(1):12-25.

9. dosReis S, Zito J, Safer D, Gardner J, Puccia K, Owens P. Multiple psychotropic medication use for youths: a two-state comparison. J Child Adolesc Psychopharmacol. 2005;15(1):68-77.

10. Olfson M, Marcus S, Weissman M, Jensen P. National trends in the use of psychotropic medications by children. J Am Acad Child Adolesc Psychiatry. 2002;41(5):514-21

11. Rappley M, Eneli I, Mullan P, et al. Patterns of psychotropic medication use in very young children with attention-deficit hyperactivity disorder. J Dev Behav Pediatr. 2002;23(1):23-30.

12. Bhatara V, Feil M, Hoagwood K, Vitiello B, Zima B. Datapoints: trends in combined pharmacotherapy with stimulants for children. Psychiatr Serv. 2002;53(3):244

13. Hashimoto Y, Uno J, Miwa T, Kurihara M, Tanifuji H, Tensho M. Effects of antipsychotic polypharmacy on side-effects and concurrent use of medications in schizophrenic outpatients. Psychiatry Clin Neurosci. 2012;66(5):405-10.

14. Kramer T. Polypharmacy. 2000. Available at: http://www.medscape.com/ viewarticle/430552. Accessed October 13, 2018

15. Protection and Advocacy. Psychiatric polypharmacy: a word of caution. 2004. Available at: http://www.disabilityrightsca.org/pubs/702001.pdf. Accessed October 13, 2018

16. Sengul M, Karadag F, Sengul C, Karakulah K, Kalkanci O, Herken H. Risk of psychotropic drug interactions in real world settings: a pilot study in patients with schizophrenia and schizoaffective disorder. Klinik Psikofarmakoloji Bulteni-Bull Clin Psychopharmacol. 2014;24(3):235.

17. Rollason V, Vogt N. Reduction of polypharmacy in the elderly. Drugs Aging. 2003;20(11):817-32

18. Marabella J. The cost of polypharmacy. 2015. Available at: http://www. pomco.com/the-cost-of-polypharmacy/. Accessed October 13, 2018.

19. Werder S, Preskorn S. Managing polypharmacy: walking the fine line between help and harm. 2003. Available at: https://www.mdedge.com/ psychiatry/article/59699/practice-management/managing-polypharmacywalking-fine-line-between-help. Accessed October 13, 2018.

20. Rambhade S, Shrivastava A, Rambhade A, Chakarborty A, Patil U. A survey on polypharmacy and use of inappropriate medications. Toxicol Int. 2012;19(1):68.

21. Colley C, Lucas L. Polypharmacy. J Gen Intern Med. 1993;8(5):278-83.

22. Fialová D, Onder G. Medication errors in elderly people: contributing factors and future perspectives. Br J Clin Pharmacol. 2009;67(6):641-45.

23. Pollack C, Weissman G, Lemke K, Hussey P, Weiner J. Patient sharing among physicians and costs of care: a network analytic approach to care coordination using claims data. J Gen Intern Med. 2012;28(3):459-65.
24. Barnett M, Landon B, O'Malley A, Keating N, Christakis N. Mapping physician networks with self-reported and administrative data. Health Serv Res. 2011:46(5):1592-609.

25. Pollack C, Lemke K, Roberts E, Weiner J. Patient sharing and quality of care. Med Care. 2015;53(4):317-23.

26. Ong M, Olson K, Cami A, et al. Provider patient-sharing networks and multiple-provider prescribing of benzodiazepines. J Gen Intern Med. 2015;31(2):164-71.

27. Constantine R, Boaz T, Tandon R. Antipsychotic polypharmacy in the treatment of children and adolescents in the fee-for-service component of a large state Medicaid program. Clin Ther. 2010;32(5):949-59.

28. Spencer D, Marshall J, Post B, et al. Psychotropic medication use and polypharmacy in children with autism spectrum disorders. Pediatrics. 2013;132(5):833-40.

29. Andersen R. National health surveys and the behavioral model of health services use. Med Care. 2008;46(7):647-53.

30. Blanchet K, James P. How to do (or not to do) ... a social network analysis in health systems research. Health Policy Plan. 2011;27(5):438-46.

31. Subcommittee on Attention-Deficit/Hyperactivity Disorder; Steering Committee on Quality Improvement and Management, Wolraich M, et al ADHD: clinical practice guideline for the diagnosis, evaluation, and treatment of attention-deficit/hyperactivity disorder in children and adolescents. Pediatrics. 2011;128(5):1007-22.

32. Pliszka S, Crismon M, Hughes C, et al. The Texas Children's Medication Algorithm Project: revision of the algorithm for pharmacotherapy of attention-deficit/hyperactivity disorder. J Am Acad Child Adolesc Psychiatry. 2006;45(6):642-57.

33. Tohen M, Chengappa K, Suppes T, et al. Efficacy of olanzapine in combination with valproate or lithium in the treatment of mania in patients partially nonresponsive to valproate or lithium monotherapy. Arch Gen Psychiatry. 2002;59(1):62.

34. Shelton R, Tollefson G, Tohen M, et al. A novel augmentation strategy for treating resistant major depression. Am J Psychiatry. 2001;158(1):131-34

35. Kowatch R, Fristad M, Birmaher B, Wagner K, Findling R, Hellander M. Treatment guidelines for children and adolescents with bipolar disorder. J Am Acad Child Adolesc Psychiatry. 2005;44(3):213-35.

36. Agency for Healthcare Research and Quality. Closing the quality gap: a critical analysis of quality improvement strategies. Publication No. 04(07)0051-7. 2007. Available at: http://www.ncbi.nlm.nih.gov/books/NBK44015/ pdf/Bookshelf_NBK44015.pdf. Accessed October 13, 2018.

37. Medhekar R, Aparasu R, Bhatara V, et al. Risk factors of psychotropic polypharmacy in the treatment of children and adolescents with psychiatric disorders. Res Social Adm Pharm. June 13, 2018 [Epub ahead of print]. Available at: https://www.sciencedirect.com/science/article/pii/ S1551741117309816?via\%3Dihub. Accessed October 15, 2018

38. Agency for Healthcare Research and Quality. Care Coordination Measures Atlas. 2014. Available at: http://www.ahrq.gov/sites/default/files/ publications/files/ccm_atlas.pdf. Accessed October 13, 2018. 


\section{APPENDIX A ICD-9-CM Codes for Identification of Mental/Behavioral Disorders}

Psychiatric Disorder

ICD-9-CM Codes

\begin{tabular}{l|l}
\hline Attention-deficit/hyperactivity disorder & 314 \\
\hline
\end{tabular}

Bipolar disorder

296.0, 296.1, 296.4, 296.5, 296.6, 296.7, 296.8, 296.9, 301.13

Depression

Anxiety disorders

$311,296.2,296.3,300.4,309.1301 .12$

Learning disorder

$313.0,301.3,308.3,309.81,300.00-300.29,309.21$

$315.0,315.1,315.2,315.3,315.4,315.5,315.6,315.8,315.9,313.83,784.69$

Adjustment disorder

Conduct disorder 309.0, 309.3, 309.4, 309.5, 309.6, 309.7, 309.9, 309.24, 309.28, 309.89

Oppositional defiant disorder 312

(313.8

\begin{tabular}{l|l}
\hline Schizophrenia & 295, 297, 298, 299.1, 299.8, 299.9
\end{tabular}

ICD-9-CM = International Classification of Diseases, Ninth Revision, Clinical Modification.

\section{APPENDIX B Psychotropic Medications}

Medication Class

Stimulants

Nonstimulants

Amphetamine, methylphenidate

Alpha agonists

Mood stabilizers

Antidepressants

Atomoxetine

Clonidine, guanfacine

Carbamazepine, divalproex, lamotrigine, oxcarbazepine, valproate, lithium

SSRIs: citalopram, escitalopram, fluoxetine, paroxetine, fluvoxamine, sertraline, vilazodone

SNRIs: venlafaxine, duloxetine

TCAs: clomipramine, amitriptyline, desipramine, doxepin, imipramine, nortriptyline

Others: mirtazapine, vortioxetine, bupropion, trazodone

\begin{tabular}{l|l} 
Antipsychotics & First generation: haloperidol, pimozide, loxapine, perphenazine, thioridazine, thiothixene, trifluperazine
\end{tabular}

Second generation: aripiprazole, olanzapine, paliperidone, risperidone, quetiapine, asenapine, iloperidone, lurasidone, ziprasidone

Others: fluphenazine, prochlorperazine

Antianxiolytics

Alprazolam, buspirone, chlordiazepoxide, clorazepate, hydroxyzine, lorazepam, diazepam, clonazepam

SNRI = selective norepinephrine reuptake inhibitor; SSRI = selective serotonin reuptake inhibitor; TCA = tricyclic antidepressant. 